\title{
A simplified technique for removal of orbital hydatid cysts
}

\author{
Gehad E Nahri
}

\section{Abstract}

A surgical technique is described for cryoextraction of the inner germinal layer of orbital hydatid cysts. The technique was adopted in two cases. It is simple and effective and causes minimal postoperative reaction or complications. There were no recurrences over a period of 24 and 18 months respectively.

Hydatid cysts of the orbit are formed round the migrated larvae of Taenia echinococcus. The disease is not rare in the Middle East and North Africa. ${ }^{1-3}$ The diagnosis of orbital hydatid cysts presents problems and requires uncertain procedures such as blind aspiration, cystography, ${ }^{2}$ or contrast orbitography. ${ }^{4}$ Even then the diagnosis was rarely achieved preoperatively..$^{1-5}$

The surgical removal of orbital hydatid cysts is frequently complicated. Cyst rupture is rather common and may result in a severe postoperative reaction anaphylaxis, incomplete removal, or secondary implantation.

\section{Patients and methods}

CASE 1

A male child aged 6 years presented in January 1987 with unilateral painless proptosis of the left eye of a few months' duration. Ultrasonography revealed the presence of a well demarcated cyst in the lateral orbit with minimal internal echoes (Figs 1, 2). A CT scan revealed a well localised cyst with radiolucent content and a regular wall

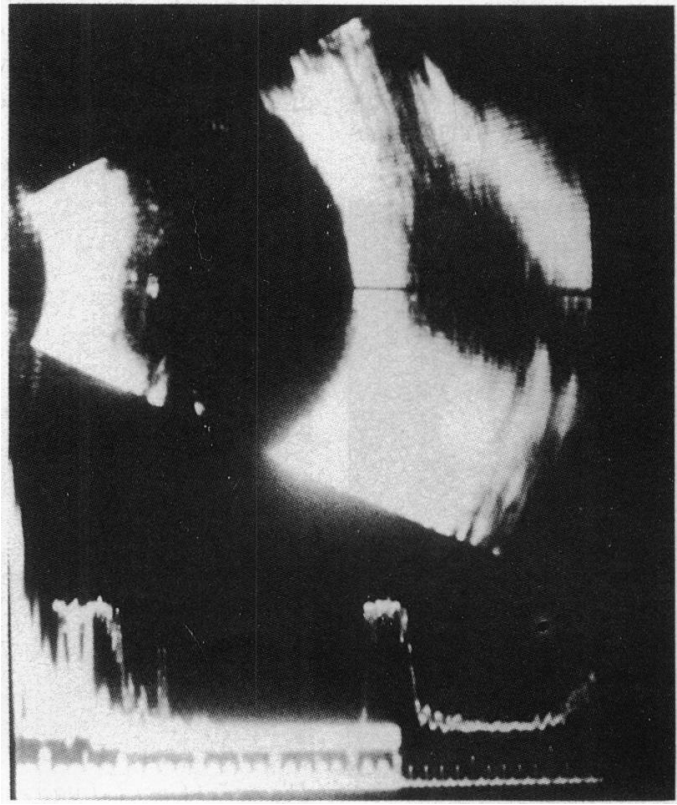

Figure 1 Case 1. Sonogram showing sonolucent orbital cyst.

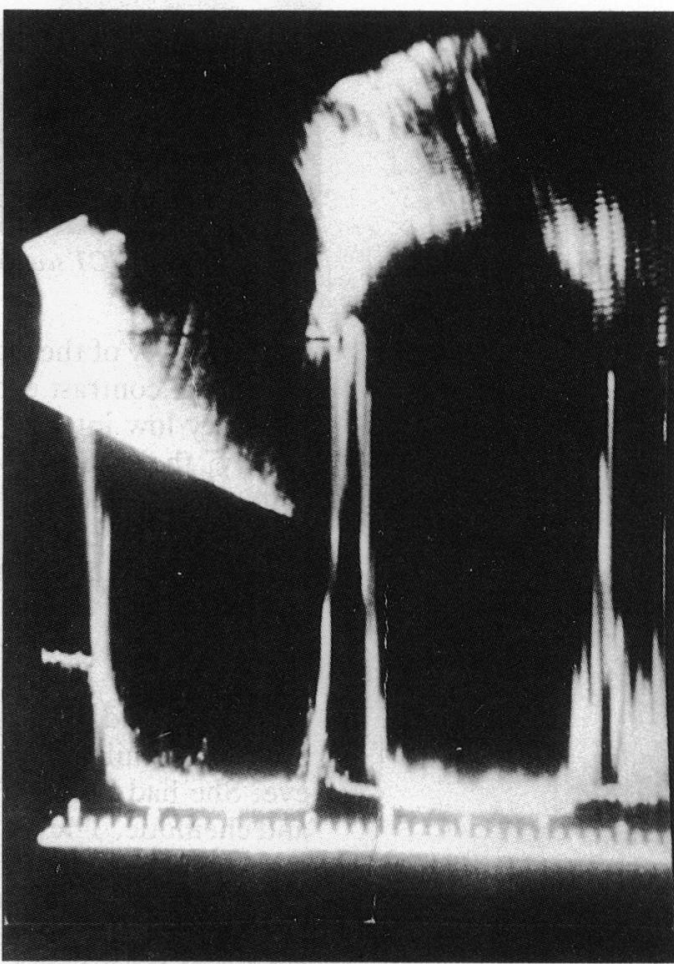

Figure 2 Case 1. Sonogram showing sonolucent orbital cyst.

that showed mild enhancement on contrast injection (Fig 3). The absence of past or present sinusitis, the lack of evidence of inflammation,

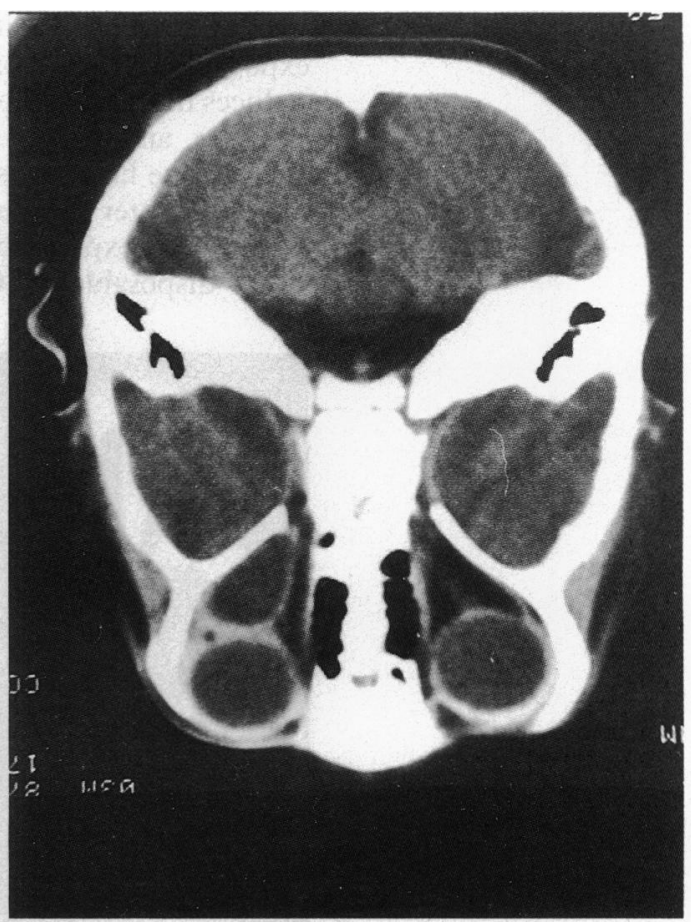

Figure 3 Case 1. CT scan showing radiolucent cyst with mild enhancement of the wall. 


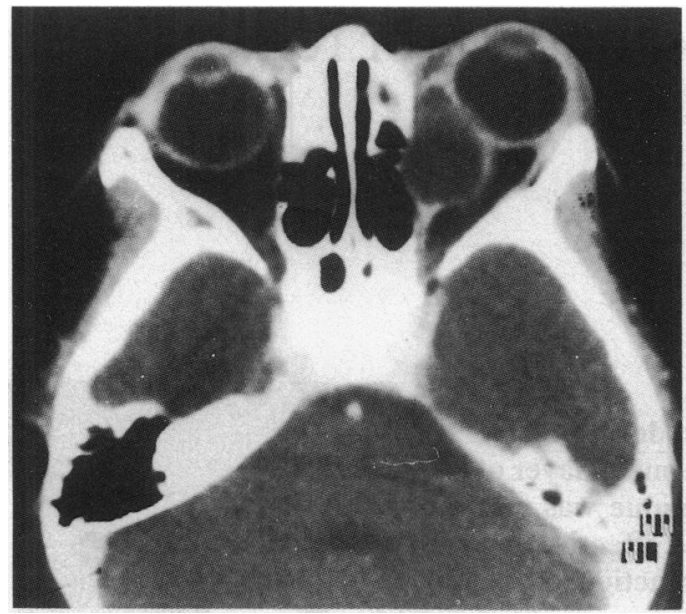

Figure 4 Case 2. CT scan appearance.

the regularity of the wall, and the mild enhancement with contrast excluded an orbital abscess. The very low internal reflectivity on ultrasonography, the complete radiolucency of the contents, and the location in the lateral orbit (which is a rare site for deep dermoids) suggested orbital hydatid cyst as the most likely diagnosis.

CASE 2

A female child aged 4 years had a few months' history of unilateral proptosis affecting her left eye. She had no pain. The conjunctiva was red and chemotic. The globe was deviated outwards, and fundus examination revealed disc oedema. CT scan was done (Figs 4, 5, 6), and the preoperative diagnosis of hydatid cyst was made from the characteristic features described above.

\section{TECHNIQUE}

The adopted technique allows the exposure and extraction of an orbital hydatid cyst without risking injury to important orbital structures. The technique lends itself to any of the surgical exposures used in orbital surgery and markedly reduces the length of orbital incisions.

Sharp and blunt dissection together with appropriate haemostasis and retraction are used until the outer fibrous wall of the hydatid cyst (ectocyst) is exposed over an area of $1 \times 1 \mathrm{~cm}$. A sharp disposable $27 \mathrm{G}$ needle on a syringe is

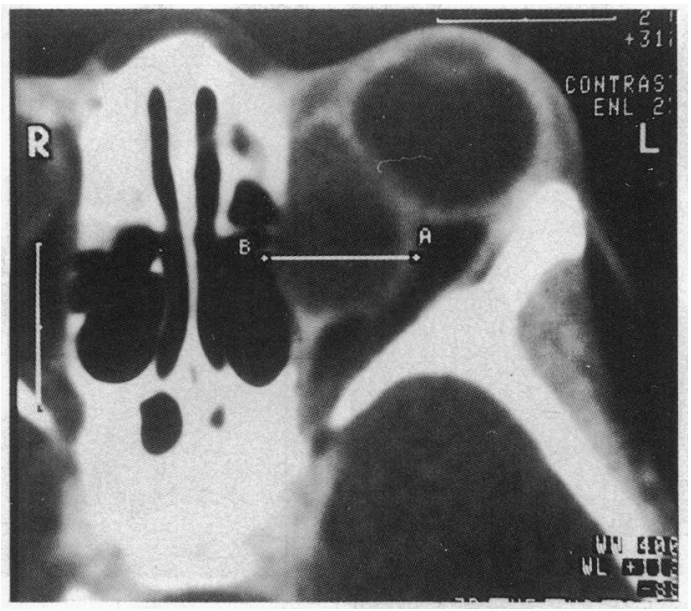

Figure 5 Case 2. CT scan appearance.

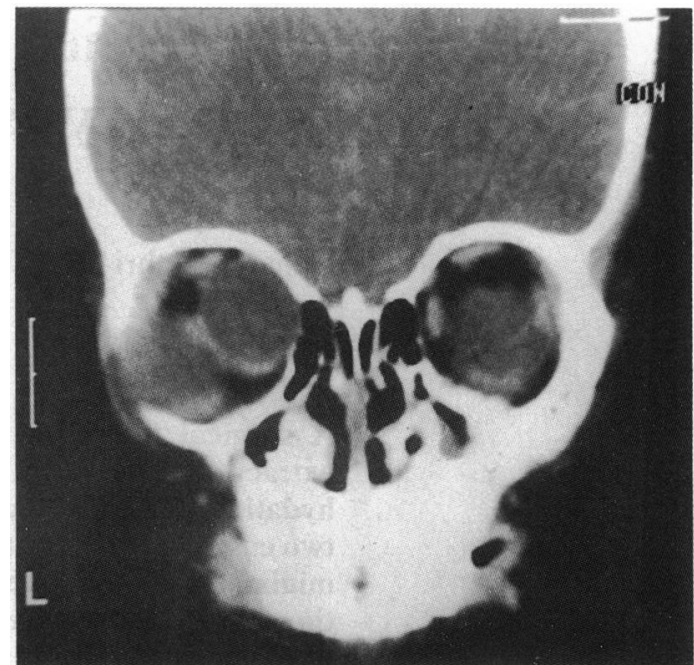

Figure 6 Case 2. CT scan appearance.

gently introduced into the cyst cavity, and as much as possible of the hydatid fluid is aspirated. The aspiration causes the inner germinal layer of the cyst (endocyst) to collapse, so that the outer fibrous wall is easily tented with a non-toothed forceps over the site of the needle puncture and snipped open. The opening is carefully enlarged for a length of $5-10 \mathrm{~mm}$ to reveal the whitish, glistening germinal layer. The endocyst is touched with an insulated cataract cryoprobe, and the probe is activated. The slippery germinative layer is easily and completely extracted through the opening (Fig 7). The outer fibrous wall is left behind, thus eliminating the necessity for extensive and frequently dangerous orbital dissection.

\section{Results}

In two cases orbital hydatid cysts were removed by employing this technique. Owing to the limited exposure needed, the transconjunctival approach was used in both cases with no difficulty.

There were no operative complications, and the postoperative reaction was minimal. There were no recurrences over a period of 24 and 18 months respectively. No postoperative enophthalmos was observed.

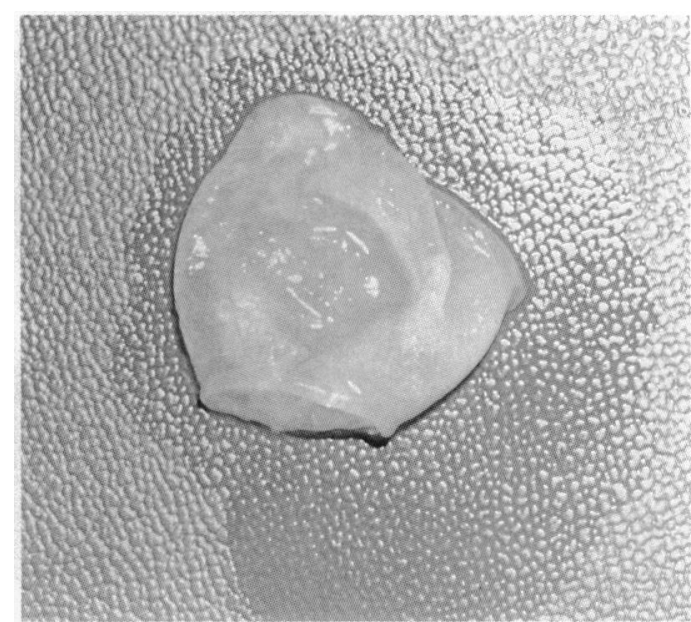

Figure 7 Case 2. Intact endocyst extracted from patient. (Natural size.) 


\section{Discussion}

Among 20 cases of proptosis in children seen in our department over the past 24 months there were two cases of hydatid cyst. Most reports on orbital hydatid cyst have concluded that a preoperative diagnosis is rarely established. These reports were of cases in which ultrasonography and CT scan were not used in reaching the diagnosis. We believe that the findings presented by these investigations may point to the diagnosis.

Surgical removal of orbital hydatid cyst is frequently complicated by cyst rupture. ${ }^{35}$ This is mostly related to the extensive dissection done on the relatively tight orbits of children and the frequently thin wall of young cysts. Intraopera- tive aspiration of the cysts presents the following advantages: confirming the preoperative diagnosis, reducing the size of the cyst, and causing the inner germinative layer to collapse so that the outer fibrous wall is safely tented and snipped open. The enophthalmos frequently observed after removal of large orbital space-occupying lesions is avoided by leaving behind the outer fibrous wall of the cyst.

1 Talib H. Orbital hydatid disease in Iraq. $B r f$ Surg 1972; 59: 391-4.

2 Handousa A. Proptosis caused by hydatid disease. $\mathrm{Br} F$ Ophthalmol 1951; 35: 607-13.

3 Baghdassarian SA, Zakharia $\mathrm{H}$. Report of three cases of hydatid

cyst of the orbit. Am F Ophthalmol $1971 ; 71: 1081-4$.
4 Mehra KS, Baneriee C, Somani PN, et al. Hydatid cyst in orbit. Acta Ophthalmol (Kbh) 1965; 43: 761-3.

5 Holland H. Hydatid of the orbit. $\mathrm{Br} \mathcal{F}$ Ophthalmol 1948; 32: 395-6. 